zusammengestellt sind. Dieser Anhang ist ganz ein Zeichen der großen Arbeitsleistung, die der Verfasser in das Buch hineingetragen hat, und die, wie ihm aufrichtig zu wünschen ist, durch einen recht guten Erfolg des Buches belobnt werden möge.

R. Grammel.

Ballistik. Von Theodor Vahien. Vereinigung wissenschaftlicher Verleger Walter de Gruyter \& Co. Berlin und Leipzig, 1922.

Gegenüber dem bekannten Handbuch der Ballistik von Cranz weist vorliegendes Buch den Vorteil auf, daß die mathematische Behandlung eingehender und systematischer durchgefuibrt ist, so besonders das Auseinanderhalten der verschiedenen mathematischen Lösungsmethoden und die Abschätzung der Genauigkeit derselben. Dabei ist auch die Fühlung mit der Praxis des Schießens immer aufrecht erhalten, das Buch ist ja, wie der Verfasser im Vorwort bemerkt, teilweise aus den Erfahrungen des Weltkrieges hervorgegangen. Das zeigt sich besonders darin, daß trotz des relativ geringen Umfanges alles sowohl praktisch als auch theoretisch Wichtige seinen Platz gefunden hat, so die Ballistik in größeren Höhen, die ja für die weittragenden modernen Geschütze von Bedeutung ist, dann die Vorgänge beim Aufschlagen des Geschosses, die Streuung, Eindringungstiefe, ferner das Zielen und Richten, die Bestimmung des Dralls usf. Hervorzuhèben ist auch eine neue Darstellung der konischen Pendelung und der Seitenabweichung der Geschasse. Dem Vorgange des Verfassers, Dimensionsbetrachtungen nicht nur als heuristisches Hilfsmittel zu verwenden, sondern damit Beweise durchzuführen, möchte ich nicht ohne weiteres zustimmen. Auch hätte vielleicht die neueste Literatur etwas mehr angeführt werden sollen, so wäre z. B. die Wienersche Methode der Reihenentwicklung zu erwähnen gewesen.

K. Wolf.

\title{
Beiträge zur streckenweisen Errechnung von Geschoßflug.
} bahnen. Von Rudolf Sängewald. Abdruck aus, den Ber. der math. phys. Klasse der sächs. Akad. d. Wissensch. zu Leipzig. LXXIII. Band, 1921.

In dieser Abhandlung gibt der Verfasser eine eingehende Durchrechnung zweier Steilflugbahnen von Geschossen auf Grund der Wienerschen Reihenmethode der streckenweisen Integration; die experimentellen Grundlagen wurden durch einen Schießversuch auf dem deutschen Artillerieschießplatz in Kummersdorf ermittelt. Als Luftwiderstandsgesetz wird das empirische von Krupp-Eberhard verwendet, dasselbe aber zuerst auf eine Tabellenform gebracht, in welcher es sich als Rechengrundiage für ein strenges Reihenverfahren eignet. Dabei wird auch ein Vergleich mit dem einheitlichen Luftwiderstandsgesetz von. Siacci. und mit dem neuesten theoretischen Ansatz von Lorenz durchgeführt, das erstere gibt eine bessere Übereinstimmung. Besonders ausführlich wird bei der Berechnung auch der Einfluß des Windes besprochen und ein Formelsystem aufgestellt, das eine stetige Bericksicbtigung eines veränderlichen Windes gestattet:

K. Wolf.

Ergebnisse der exakten Naturwissenschaften. Herausgegeben von derSchriftleitung der » Naturwissenschaften «.Verlag von J.Springer, Berlin. Erster Band 1922 mit 35 Abb. 403 S. GZ. 12, 5, geb. 14. Zweiter Band 1923 mit 38 Abb. 252. S. GZ. 8, geb. 9, 5.

Das Werk ist als ein Jahrbuch gedacht, das die wichtigsten Forschungsergebnisse in Form kleiner Monographien behandeln soll. Es wird im allgemeinen über das berichtet, was im vorangegangenen Jahre zu besonderer Bedeutung gelangt ist; der erste Band greift naturgemäß etwas weiter zurück. Da es sich nicht um eine Referatensammlung nach Art der physikalischen Berichte handeft, wird auf Vollständigkeit kein Anspruch erhoben, dafür konnten die einzelnen Artikel breit genug angelegt werden, um auch den Nichtspezialisten auf dem betreffenden Gebiete einen sehr guten Einblick in den Gegenstand zu gewähren. Inhalt des ersten Bandes: 